Jhon Alexander Méndez Sayago Jhon.mendez@correounivalle.edu.co Calle 13 No. 100 - 00. Universidad del Valle, Ciudadela Universitaria Meléndez, Cali - Colombia.

Máster en Economía (Universidad Javeriana) y Economía Ambiental (Universidad de los Andes). Ingeniero Civil, UFPS. Profesor Asistente, Departamento de Economía, Investigador Grupo GERA, Universidad del Valle. Cali - Colombia.

Hugo Alfonso Hernández Escolar hahernandeze@ulibertadores.edu.co Cra. 16 \# 63A - 68, Bogotá - Colombia.

Master en Economía, Universidad Javeriana. Economista,

Universidad Nacional. Profesor investigador de la Universidad los Libertadores, Bogotá - Colombia.

Nubia Stella Carreño nubiacarreño@ulibertadores.edu.co Cra. 16 \# 63A-68, Bogotá - Colombia.

Economista, Universidad la Gran Colombia. Profesora de la Universidad los Libertadores- Bogotá.

Artículo de investigación científica y tecnológica Según Clasificación Colciencias

Fecha de recepción: agosto 25 de 2011 Fecha de corrección: octubre 24 de 2011 Fecha de aprobación: diciembre 20 de 2011

\section{Evaluación del impacto de las Microfinanzas sobre los ingresos y la generación de empleo en Colombia*}

Evaluation of the impact of microfinance on revenue and the generation of employment in Colombia

Évaluation de l'impact des micro-finances sur les revenus et la création d'emploi en Colombie

\section{Resumen}

El artículo evalúa la capacidad de las herramientas microfinancieras para impulsar el mejoramiento de ingresos de las pequeñas y medianas unidades productivas en Colombia, así como la creación de puestos de trabajo en este segmento empresarial. Para tal cometido, se efectúa una estimación de los impactos del crédito formal, de cualquier tipo de crédito, y del crédito otorgado por la participación en programas de apoyo empresarial como FOMIPYME. Las técnicas para la estimación de los impactos de los tratamientos (microcrédito) comprendieron: i) los estimadores Matching no paramétricos del vecino más cercano y Kernel; ii) el estimador de variables instrumentales; y iii) el estimador del efecto local promedio del tratamiento (LATE). En todas las mediciones de los efectos del tratamiento se aceptó la hipótesis nula de que el microcrédito no tiene impacto positivo sobre las ganancias y la generación de empleo de las Micro, Medianas y Pequeñas Empresas.

Palabras clave: microfinanzas, evaluación de impacto, estimador Matching, variables instrumentales, estimador LATE.

\footnotetext{
* Este artículo de investigación es resultado del proyecto "Evaluación del impacto de Políticas contra el desempleo y la Pobreza: Caso del Microcrédito en la ciudad de Bogotá", Financiado por la Universidad los Libertadores, Bogotá, Colombia.
} 


\section{Evaluation of the impact of microfinance on revenue and the generation of employment in Colombia}

Evaluación del impacto de las Microfinanzas sobre los ingresos y la generación de empleo en Colombia

Évaluation de l'impact des microfinances sur les revenus et la création d'emploi en Colombie

\section{Abstract}

The article evaluates the capability of the micro-financial tools to promote revenue improvement in small and medium-sized productive units in Colombia, as well as the creation of jobs in this business segment. For said purpose, estimation was made of the impact of formal credit, of any type of credit, and the credit awarded for participating in entrepreneurial support programs like FOMIPYME. The techniques for the estimation of impact of the treatments (microcredit) comprised: i) the Matching non-parametric estimators of the nearest neighbor and Kernel; ii) the estimator of instrumental variables; and iii) the effect of the local average treatment estimator (LATE). In all the measurements of the effects of the treatment, the null hypothesis was accepted that microcredit does not bear a positive effect on profits and the generation of employment by Micro, Small, and Mediumsized Enterprises.

Keywords: micro-finance, evaluation of impact, matching Estimator, instrumental Variables, LATE estimator.
Évaluation de l“impact des microfinances sur les revenus et la création d'emploi en Colombie

\author{
Evaluación del impacto de las \\ Microfinanzas sobre los ingresos y la \\ generación de empleo en Colombia
}

Evaluation of the impact of microfinance on revenue and the generation of employment in Colombia

\section{Résumée}

Ĺarticle propose une évaluation de la capacité des outils micro-financiers pour renforcer l'accroissement des revenus des petites et moyennes unités productives en Colombie, ainsi que la création de nouveaux postes de travail dans ce secteur. Cette évaluation repose sur une estimation des impacts du crédit formel, du crédit reçu par la participation dans des programmes de soutien aux entreprises tel que FOMYPIME, et tout autre type de crédit. Les techniques pour l'estimation des impacts des conditions (micro-crédit) ont compris: i) les estimateurs Matching non paramétriques du voisin le plus proche et Kernel; ii) l'estimateur de variables instrumentales, et iii) l'estimateur de l'effet local moyen des conditions (LATE). Pour toutes les méditions des effets des conditions on a accepté l'hypothèse nulle selon laquelle le micro-crédit n'a pas d`impact positif sur les revenus et la création d'emploi dans les micros, moyennes et petites entreprises.

Mots clef: micro-finance, évaluation d’impact, estimateur Matching, variables instrumentales, estimateur LATE. 


\section{Evaluación del impacto de las Microfinanzas sobre los ingresos y la generación de empleo en Colombia}

\section{Introducción}

El microcrédito es una modalidad de financiamiento en expansión gracias a su reputación como herramienta eficiente para reducir los niveles de carencia de capital productivo por parte de la población. La experiencia exitosa del Banco Grameen en Bangladesh, como indica Marbán (2005), ha convertido a las microfinanzas en una de las herramientas más populares de fomento para el desarrollo productivo.

La teoría del microcrédito expone que éste favorece la consecución de beneficios porque, al mejorar el acceso al crédito, aumenta el nivel de capital y se fomenta en los hogares la disposición a crear una empresa o a expandirla si ésta ya existe. Uno de los estudios más recientes y completos acerca de las microfinanzas, efectuado por el Banco Mundial, recopila abundante material empírico que favorece la tesis de que la falta de acceso a las finanzas es un mecanismo crítico para perpetuar la desigualdad del ingreso, y mantener un bajo crecimiento (Larraín, 2009, p. 5).

En América Latina, dentro de la literatura existente sobre la evaluación del impacto del microcrédito, se encuentran los estudios de Aroca (2002), Henríquez (2009) y Cotler y Rodríguez (2009).

En Aroca (2002) se evaluó el impacto de programas de microcrédito de bancos y Organizaciones no Gubernamentales brasileñas y chilenas, sobre el ingreso promedio de las personas que recibieron microcrédito, mediante la técnica propensity score y el estimador Matching. Los resultados fueron ambiguos: altamente positivo para el microcrédito administrado por los bancos en Brasil; una evidencia débil para el mismo caso en Chile y provenientes de ONG en Brasil; e impacto negativo en Chile, para el microcrédito proveniente de ONG.

Henríquez (2009) mide el impacto de múltiples dosis de microcrédito, sobre las variaciones de las ventas, el empleo y la formalidad de las microempresas en Chile, utilizando la metodología de Diferencias en Diferencias Matching. Se concluye que las microempresas que hicieron uso intensivo del crédito, obtienen efectos deseables sobre el desempeño de sus firmas. Las empresas que obtuvieron quince o más créditos, aumentaron sus ventas en un $23 \%$, y un $14 \%$ de éstas se incorporó al sistema formal. Cuando el número de créditos obtenidos estuvo entre diez y catorce, el incremento en las ventas asciende al $26 \%$. Las microempresas que obtuvieron entre cinco a nueve microcréditos redujeron en menor proporción su contratación (38\%), versus aquellas empresas que demandan cero créditos en el período. Las microempresas con menos de 5 créditos no presentaron efectos sobre la evolución de su desempeño medido en ventas, empleo o formalidad.

Desde otro enfoque, Cotler y Rodríguez (2009), evalúan el impacto del microcrédito sobre un índice de propiedad de activos físicos de los hogares, utilizando la técnica propensity score y el estimador Matching, asumiendo que la tenencia de activos físicos, puede considerarse un buen indicador del bienestar del hogar y de su capacidad para mejorar sus condiciones socioeconómicas. Se concluye que el financiamiento formal tiene efecto positivo en el caso urbano y nulo en el caso rural.

Mediante el presente trabajo se pretende replicar el empleo de técnicas de tratamiento para la evaluación de impacto de las microfinanzas sobre la población de bajos ingresos en Colombia, utilizando la información disponible en la base de datos 2005-2009 de las convocatorias efectuadas por el Fondo Colombiano de Modernización y Desarrollo Tecnológico de las Micro, Pequeñas y Medianas Empresas (FOMIPYME). Se evalúan los impactos del crédito formal, de cualquier tipo de crédito, y del crédito derivado de la participación en el programa de FOMIPYME, sobre las ganancias y la generación de empleo de las micro, medianas y pequeñas empresas (MIPYME). La estimación del efecto del tratamiento se realiza mediante técnicas estadísticas y econométricas que hacen uso del supuesto de selección sobre observables y selección sobre componentes no observados. 


\section{Evolución reciente de las microfinanzas en Colombia}

A pesar de la continua promoción y difusión de iniciativas de financiamiento para las capas más pobres de la población en muchas regiones del mundo, el concepto de microfinanzas no se halla delimitado. No obstante ello, existe una serie de elementos básicos que ayudan a los analistas económicos a trazar una línea separadora entre el financiamiento preponderante en la economía y un financiamiento alternativo que presenta diferencias cualitativas frente al primero.

Por ejemplo, Mena (2001), citada por Aristizábal (2007), concibe a las microfinanzas como servicios financieros prestados a hogares de bajos ingresos mediante condiciones distintas a las de la banca convencional, haciendo especial alusión a las garantías reales; Barona (2003) expone, de manera más sintética, que las microfinanzas son enfoques nuevos para financiar a los pobres; $y$ Arango (2008) las define como programas de asistencia a las medianas y pequeñas empresas, con el propósito de ofrecer posibilidades de superación de la pobreza.

Sea cual fuere la definición más aceptable para propósitos de estudio o de diseño de políticas -no necesariamente públicas, sino también de las entidades financiadoras, como ONG, cooperativas - la propia banca-, cabe entender que existen dos formas diferenciadas de financiamiento: una soportada en garantías e información del cliente, verificables casi en tiempo real, asociadas a volúmenes altos de crédito individual y plazos prolongados para la devolución de los recursos; y otra cuya base de confianza modifica o suple las garantías, el nivel de conocimiento inicial del cliente es precario y los recursos se entregan en cuantías bajas y a corto plazo.

En este segundo mercado o espacio transaccional se ha venido descubriendo un alto potencial de crecimiento, indicando con ello que las reglas estrictas del financiamiento tradicional generaron durante muchas décadas una brecha entre quienes podían acceder al crédito formal y quienes debían recurrir a un mercado negro. La esencia del microfinanciamiento es entonces la evolución institucional encaminada a formalizar el crédito y otros servicios financieros de que pueden disponer hoy en día las unidades económicas que por su situación de informalidad y/o pobreza no pueden acceder al financiamiento corriente.
Como detrás de las unidades económicas hay familias, muchas en condiciones de vulnerabilidad, los analistas de las microfinanzas frecuentemente enlazan el tema del crédito a los pequeños emprendedores con el de la superación de la pobreza y el subdesarrollo, pero es preciso adentrarse en la mecánica de las operaciones financieras para justificar y estimar este vínculo.

Desde una perspectiva histórica, el crédito a pequeños empresarios es casi paralelo al refinamiento del sector bancario. Cárdenas (2004), citado por Arango (2008) menciona que puede hablarse de una banca microempresarial desde el siglo XVII. En el siglo XIX tiene un papel muy activo en este tipo de financiamiento el cooperativismo naciente. Pero sólo hasta la segunda mitad del siglo $X X$ se perfila lo que va a convertirse en una ola incesante de cambios en el mercado financiero. La experiencia inspiradora del Grameen Bank, ideado por Muhammad Yunus y orientado con especial énfasis a las mujeres, ha tomado distintos matices en todas las latitudes del tercer mundo, aunque con dos zonas de especial posicionamiento: el sur de Asia y América Latina. En la primera región se destacan las realizaciones del Banco para la Agricultura y las Cooperativas Agrícolas (Tailandia) y los bancos indonesios Rakyat y Bada Credit Kecamatan (Norton, 2004, citado por Arango, 2008). En Latinoamérica son diversos los ejemplos de instituciones pioneras: Banco Sol (Bolivia), la Financiera Compartamos (México) y la Fundación Grameen, presente en varios países y con énfasis particular en el financiamiento rural.

El posicionamiento de Colombia en la actividad microfinanciera latinoamericana, según el último reporte de 2009 del Microfinance Information Exchange (MIX), es intermedio. En lo que respecta al número de instituciones detectadas en el mercado microfinanciero, fueron registradas 48 , frente a 100 de Ecuador y 89 de Perú; y en cuanto a la penetración dentro de la población pobre, el indicador es de $6,5 \%$, igualando el promedio de Suramérica. En el número de prestatarios por institución microfinanciera (IMF), Colombia logra la cifra más alta de toda América Latina (39.701), pero se viene presentando una muy alta concentración en el universo de clientes, pues las cinco mayores IMF abarcan el $88,8 \%$ de los prestatarios. Si no se incluyen estas 
entidades, el promedio se reduce a 8.687 usuarios por IMF (MIX, 2009).

Barona (2004), citando a Kirkpatrick y Maimbo (2002), identifica tres etapas en el desarrollo institucional de las microfinanzas en nuestro país. La primera, desde 1930 hasta los años 80, se caracterizó por el énfasis dado en la política pública al sector rural, al cual se dedicaron esfuerzos notables en materia de crédito subsidiado. Con el paso del tiempo, la principal entidad en este esquema de apoyo, la Caja Agraria, colapsó a finales de los noventa por la mala selección de clientes, la coyuntura económica y prácticas corruptas. Su sucesor, el Banco Agrario, de mucha menor escala, fue creado con una mayor afinidad misional a la banca convencional.

La segunda etapa es la que recibe propiamente los conceptos de Yunus sobre la capacidad de las capas pobres de la población para responder por sus obligaciones, aun sin garantías otorgadas. En Colombia este enfoque privilegió la localización de clientes en entornos urbanos, y tuvo como su iniciativa más notable el Programa de Crédito para la Microempresa, con el respaldo del Banco Interamericano de Desarrollo (BID). En la esfera pública, cabe resaltar el papel del Departamento Nacional de Planeación (DNP) en materia de análisis técnico y el Instituto de Fomento Industrial como intermediador de recursos (banco de segundo piso, a modo de respaldo ante la banca comercial). Las organizaciones privadas protagonistas en esta etapa, hasta mediados de los noventa, fueron Finamérica, la Fundación Santo Domingo y la Fundación WWB Bank (Banco de la Mujer) de Cali.

La tercera y actual fase data desde finales de los noventa, y tiene origen en los pronunciamientos de algunos críticos como Hulme, Rutherford, Wrigth y Matin, citados por Barona (2004). Para ellos, la presión por autosostenerse económicamente ha llevado a muchas IMF a reclasificar al alza su perfil de cliente, es decir, a desechar como prospectos de crédito a los emprendedores de menos recursos. Otra característica de este período es el interés de extender la esfera microfinanciera a los servicios de ahorro, seguros y pensiones, entre otros complementos al crédito. Para fortalecer esta estrategia integral adquiere mayor pertinencia la regulación provista por el sector público, y se espera un especial protagonismo del sector cooperativo, fuertemente golpeado por la crisis financiera de los noventa, pero el cual mantiene una fuerte presencia en el escenario rural y entre el empresariado de menor escala.

El futuro del microcrédito como herramienta de financiamiento atractiva tanto para las instituciones otorgantes como para los clientes, tiene sustento en sus distintos indicadores. Respecto de la cartera bruta total de los establecimientos de crédito, la participación de los microcréditos se ha elevado considerablemente, de $1,7 \%$ al cierre de 2007 a 2,8\% a finales de 2009 (ver gráfico 1). En valor absoluto las cantidades colocadas siguen siendo muy reducidas respecto al total de operaciones de la banca, pero el crecimiento sostenido da cuenta de un amplio espectro de clientes potenciales por alcanzar.

Muy positivo también ha sido el desempeño de la calidad de cartera, si se considera que en la coyuntura previa a la crisis hipotecaria norteamericana y su contagio al sistema financiero europeo, paralela a su vez a la abrupta disminución del comercio con Venezuela, la cartera vencida venía creciendo como proporción de la cartera bruta (gráfico 2). No obstante, este patrón de comportamiento alcanzó su punto cumbre en marzo de 2008, con un indicador de $8,1 \%$, y desde allí se presentó una rápida mejoría, hasta retornar al cierre de 2009 a niveles próximos a los de comienzos de 2007, con un indicador de 5,7\% (Gráfico 1 y Gráfico 2).

Otro referente, como lo es el cubrimiento de cartera vencida (provisiones / cartera vencida), ha mostrado una mejoría ostensible desde finales de 2008 , pasando de $60 \%$ en diciembre de 2008 a más de $70 \%$ al cierre de 2009 (gráfico 2). En los dos años anteriores los niveles se habían mantenido muy estables. Una mayor provisión o reserva por cada peso de cartera vencida permite mayor sostenibilidad para la entidad otorgante en el caso de dificultades en el repago de los préstamos.

Al comparar los indicadores anteriores con los obtenidos por el agregado de establecimientos crediticios (gráficos 3 y 4), se reafirma la ventaja del microcrédito en lo que atañe a la calidad de la cartera, si bien el valor de tal ventaja ha venido reduciéndose ligeramente. Al concluir 2009, los indicadores fueron de 4,1\% para el microcrédito y $5,7 \%$ para el total de establecimientos. 


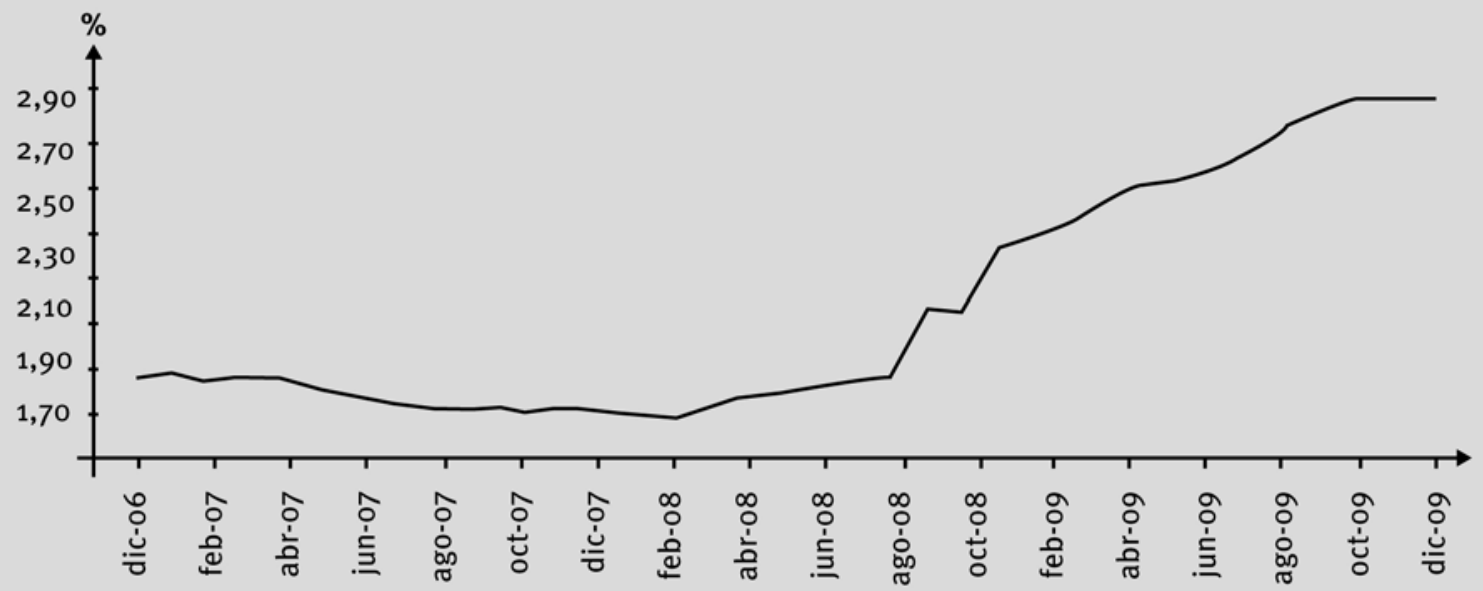

Fuente: Superintendencia Financiera de Colombia, página web. Cálculos propios.

\section{Gráfico 2. Microcrédito - Indicadores de calidad de cartera y cubrimiento}

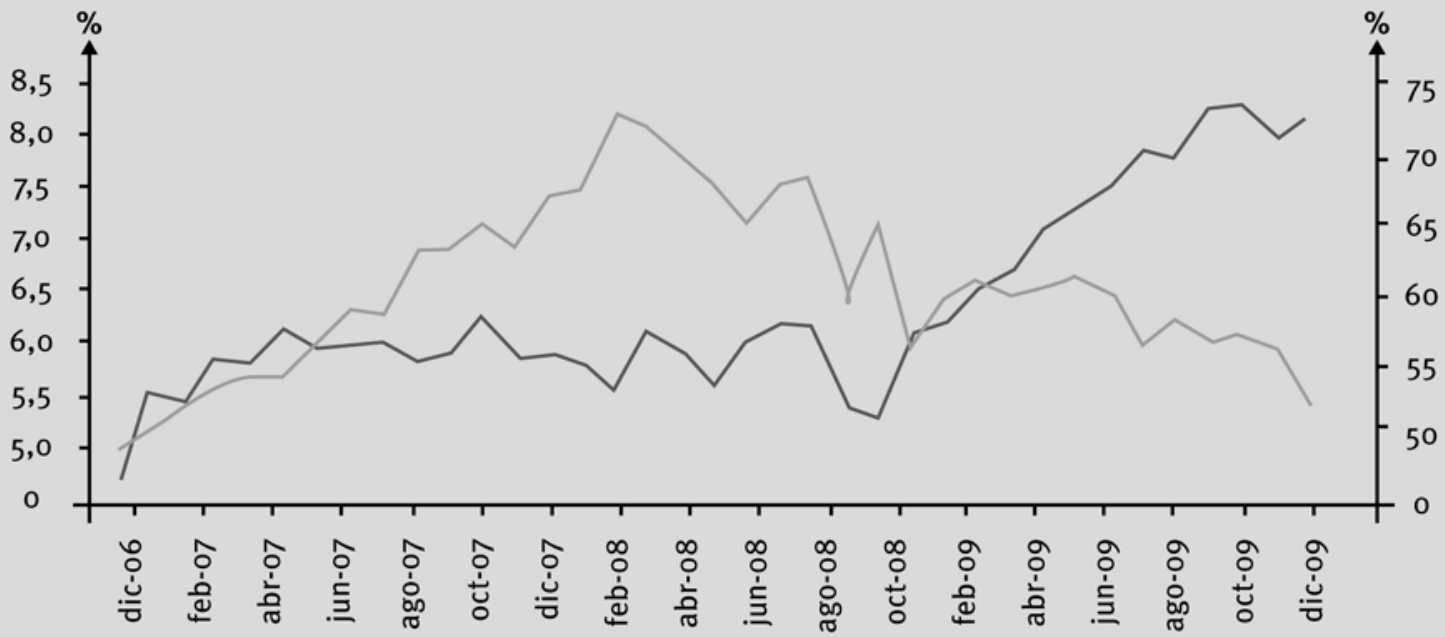

- Calidad de Cartera

- Cubrimiento

Nota: la calidad de cartera se mide en el eje izquierdo, y el indicador de cubrimiento en el eje derecho

Fuente: Superintendencia Financiera de Colombia.

Por su parte, el cubrimiento de cartera vencida (gráfico 4) posee un indicador superior para el agregado de establecimientos de crédito en razón a los mayores plazos propios de las operaciones de financiamiento convencional, especialmente en lo referente al crédito hipotecario. A pesar de ello, la estabilidad de esta variable en las operaciones de microcrédito es señal de que no han sido requeridos ajustes en el tiempo, y si la provisión es menor, se dispone de recursos adicionales para expandir los créditos (Gráfico 3 y Gráfico 4).

Al considerar la evolución conjunta de las tasas de interés y los desembolsos mensuales de microcréditos en los últimos dos años, el gráfico 5 enseña que no hay una orientación definida para la variación conjunta, puesto que la correlación entre las dos series es muy cercana a cero $(-0,22$ descontando la observación atípica que aparece más alejada en la figura). También se encuentra que a la vez que el intervalo de variación de las tasas es estrecho, entre $30 \%$ y $32 \%$ efectivo anual, no ocurre lo mismo con los desembolsos, cuyo valor oscila en un rango muy amplio. En apariencia, el microcrédito tiene una mayor adaptabilidad ante pequeños cambios en el entorno económico, y en consecuencia los desembolsos llevan una senda irregular, distinta a la de créditos a mayor plazo que están menos supeditados a la coyuntura específica de la economía (Gráfica 5 ) 

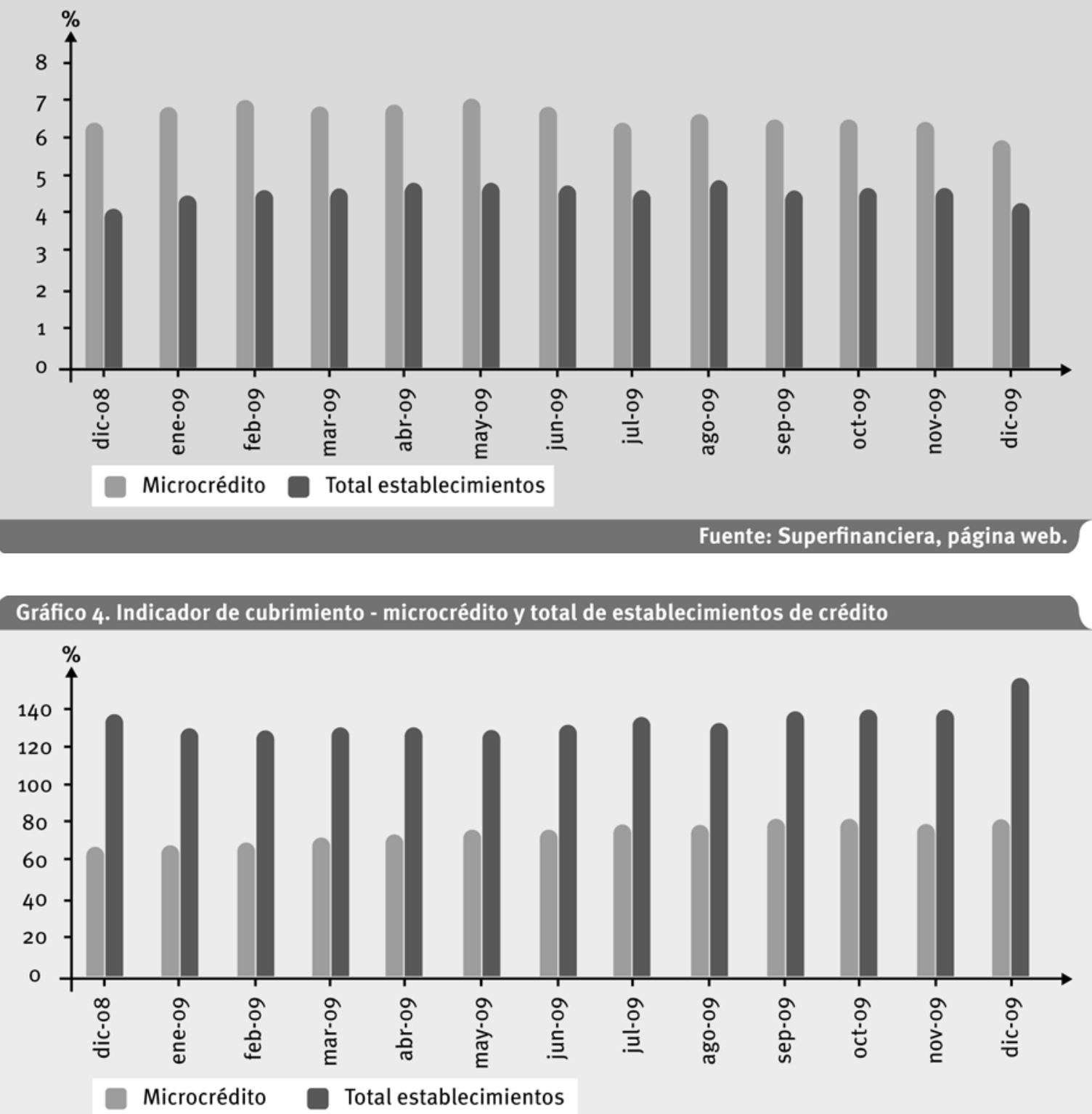

En los últimos años, la política pública de apoyo al emprendimiento tuvo una de sus manifestaciones más visibles en el programa Banca de las Oportunidades. Este se ha concebido como un esquema de articulación de entidades de distinta naturaleza para crear una red institucional financiera que impulse el proceso de bancarización de segmentos de población que tradicionalmente utilizan mecanismos extrabancarios de ahorro y crédito.

Las dos estadísticas de resultado más representativas del programa corresponden a los corresponsales no bancarios y los desembolsos de microcrédito. En cuanto al primer ítem, al cierre de septiembre de 2010 se llegó a 9.458 corresponsales en cerca de 800 municipios. Los desembolsos de recursos a microempresas en el cuatrienio agosto 2006 - agosto 2010 alcanzaron $\$ 18$ billones en 6,2 millones de operaciones (es decir, algo menos de \$3 millones en promedio por crédito). Cabe distinguir en especial a los microempresarios que recibieron crédito por primera vez (de manera formal), que suman para el período mencionado 1,7 billones, de los cuales más del $60 \%$ tramitó la operación de crédito ante una $\mathrm{ONG}^{1}$. 


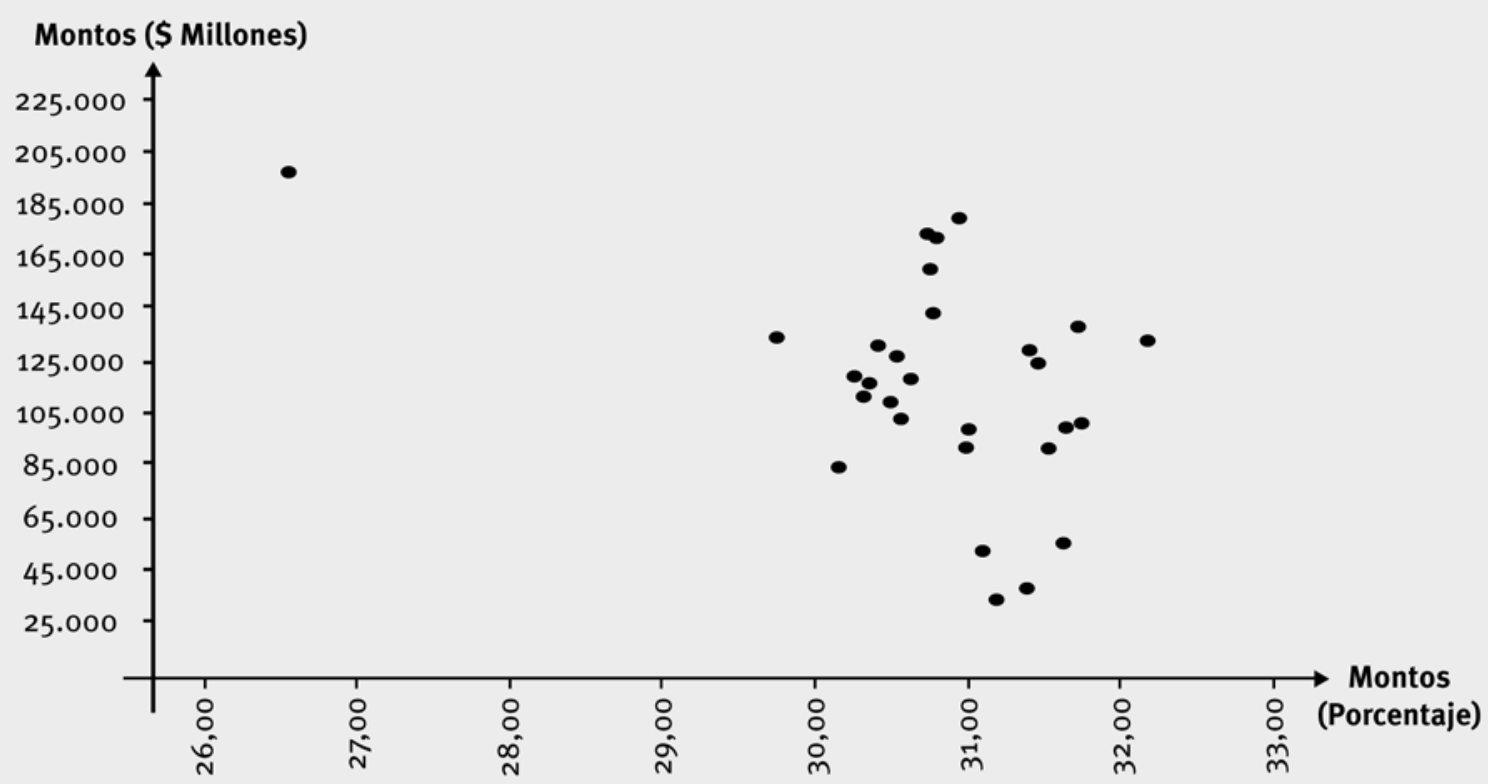

Fuente: Superfinanciera.

\section{Técnicas para la evaluación del impacto del microcrédito}

La evaluación del impacto de una política pública o programa como el microcrédito, está destinada a determinar si la intervención tuvo los efectos deseados sobre los individuos, y si esos efectos son atribuibles al programa. Esta evaluación se realiza mediante inferencia causal, que consiste en determinar los efectos de la intervención sobre algunas variables relevantes relacionadas con la política.

El efecto causal del tratamiento sobre un individuo $\mathrm{i}$, en términos de la variable y sobre la cual se espera que la política tenga un impacto significativo será:

$\delta_{i}=y_{1 i}-y_{0 i}$

$\mathrm{V}_{1 i}$ : Valor de la variable de interés si el individuo $i$ participa en el programa.

$\mathrm{Y}_{o i}:$ Valor de la variable de interés si el individuo $i$ no participa en el programa.

El hecho de que un mismo individuo no pueda recibir y no recibir tratamiento al mismo tiempo, complica la tarea de medición del efecto de la política. Este hecho es denominado problema fundamental de los estudios de causalidad ${ }^{2}$ (Holland, 1986). Para superar esta complicación, las técnicas para la evaluación del impacto de políticas públicas hacen uso de dos grupos. Un grupo afectado por el tratamiento (grupo de tratados) y un grupo de no tratados (grupo de control).

La medida más utilizada para estimar de una política pública o programa, cuando el programa no es asignado aleatoriamente entre la población, sino que tiene como objetivo cierto tipo de individuos, es el efecto promedio del tratamiento sobre los tratados (ATT). ATT se define como el efecto promedio del tratamiento en las personas participantes en el programa por evaluar (Blundell y Costa, 2007).

$$
\mathrm{ATT}=E\left(\gamma_{1}-\gamma_{-} 0 \mathrm{ID}=1\right)(2)
$$

Siendo $\mathrm{D}$ la variable indicadora de tratamiento:

$D=1$ Si i participa en el programa (recibe microcrédito)

$D=0$ Si i no participa en el programa (no recibe microcrédito)

Adviértase lo siguiente:

- Con la información disponible del grupo de tratados $y_{1 i} \forall_{i \in C}$ se obtiene $E\left(\gamma_{1} \mid D={ }_{1}\right)$.

- Con la información disponible del grupo de tratados $y_{0 i} \forall_{i \in 0}$ se obtiene $E\left(\gamma_{1} \mid D==_{1}\right)$. 
Haciendo uso de la información disponible, se puede estimar el efecto del tratamiento $\Delta$ a partir de la expresión:

$$
\Delta=E\left(\gamma_{1} \mid D=1\right)-E\left(\gamma_{0} \mid D=0\right)(3)
$$

El estimador del efecto del tratamiento $\Delta$ calculado a partir de la información disponible puede ser un estimador sesgado de ATT. Este sesgo es denominado sesgo de selección:

$$
\begin{gathered}
\Delta=E(\underbrace{\left.y_{1} \mid D=1\right)-E\left(y_{0} \mid D=1\right)+}_{A T T} \\
\underbrace{\left.E\left(y_{0} \mid D=1\right)-E\left(y_{0} \mid D=0\right)\right\}}_{\text {Sesgo de selección }}
\end{gathered}
$$

\section{Sesgo de selección}

"El problema de sesgo de selección en la evaluación de impactos es causado por el hecho de que los individuos que pertenecen a dos grupos distintos (como participantes del programa y no participantes) tienen determinadas características que no pueden ser observadas por el evaluador y afectan tanto a la decisión de participar en el programa así como a sus resultados (por ejemplo, la capacidad o la motivación de una determinada persona, relaciones familiares)" (Moral, 2009, pág. 7)

Sin embargo, si las características que afectan la elegibilidad del tratamiento y sus resultados son observadas, se puede bajo el supuesto de selección sobre los observables, asumir la ignorabilidad del tratamiento3 ${ }^{3}$ Rosenbaum y Rubin, 1983).

La ignorabilidad del tratamiento se mantiene si el tratamiento $D$ es una función determinística de $X$, lo cual es denominado selección sobre los observables (Wooldridge, 2002). El supuesto de ignorabilidad del tratamiento implica que:

$$
y_{1}, y_{0} \perp \mathrm{D} \mid \mathrm{X} \Rightarrow\left[\begin{array}{l}
E\left(y_{1} \mid D, X\right)=E\left(y_{1} \mid X\right) \\
E\left(y_{0} \mid D, X\right)=E\left(y_{0} \mid X\right)
\end{array}\right.
$$

Condicional a $\mathrm{X}$, el efecto promedio del tratamiento sobre los tratados será:

$$
\begin{gathered}
A T T(X)=E\left(y_{1}-y_{0} \mid D=1, X\right)= \\
E\left(y_{1} \mid D=1, X\right)-E\left(y_{0} \mid D=1, X\right)
\end{gathered}
$$

Como se comentó anteriormente, $\mathrm{E}\left(\mathrm{\gamma}_{0} \mid D=1, X\right)$ no se puede hallar a partir de la muestra porque falta la evidencia contrafactual para los individuos tratados, pero bajo el supuesto de selección sobre los observables se tiene que:

$$
\mathrm{E}\left(\mathrm{\gamma}_{0} \mid D=1, \mathrm{X}\right)=\mathrm{E}\left(\mathrm{y}_{0} \mid D=0, \mathrm{X}\right)(5)
$$

Así que la estimación del efecto promedio del tratamiento sobre los tratados, haciendo uso de $E\left(\gamma_{0} \mid d=0, X\right)$ en lugar de $E\left(\gamma_{0} \mid d=1, X\right)$ no generará ningún sesgo:

$$
A T T(X)=E\left(y_{1} \mid D=1, X\right)-E\left(y_{0} \mid D=0, X\right)
$$

El supuesto de ignorabilidad del tratamiento se cumple en la realización de un experimento social, donde la admisión al programa dependa de determinadas variables de certificación $X$ y luego la asignación del tratamiento sea aleatoria. No obstante, aunque no se realice un experimento de esta naturaleza, bajo el supuesto de selección sobre los observables, se pueden generar grupos de comparación que sean similares al grupo de control de un experimento aleatorio, lo cual garantiza que se cumpla la igualdad (5).

\subsection{Estimador Matching}

El emparejamiento estadístico ofrece una forma de construir un grupo de control lo más similar posible al grupo de tratados, en términos de las características observables antes de asignado el tratamiento. "El objetivo de la técnica de Matching es el de emparejar cada participante, con aquellos no participantes similares a él, simulando así un experimento aleatorio" (Moral, 2009, pág. 21). Lo interesante del método de Matching es que ofrece una medida objetiva, porque la técnica de estimación emplea un método riguroso para elegir las personas comparables a las tratadas (Aedo y González, 2002).

El problema del emparejamiento estadístico es que para la construcción del grupo de control, se deben encontrar individuos no tratados que sean similares a los individuos tratados, en términos de los condicionantes $X$ bajo cierta métrica. Si el vector $X$ está compuesto por muchas variables, resulta difícil definir en la práctica el grado de cercanía entre dos individuos según 
los valores de sus variables en $X$. Es decir, existe un problema de dimensionalidad. Esto dificultó en principio, la formación de grupos de control adecuados, perjudicando cualquier evaluación de programas sociales.

Para reducir la complejidad del condicionamiento por las variables en X, Dehejia y Wahba (1999) propusieron el uso del propensity score, o probabilidad de ser sujeto de tratamiento, para reflejar cualquier diferencia en las variables $X$. El objetivo es obtener una medida para la comparabilidad de los individuos del grupo de tratados y del grupo de control; en términos de tener valores de propensity score más cercanos.

Rosembaum y Rubin (1983) demostraron se obtienen los mismos resultados, si en vez de condicionar las distribuciones de la variable de resultados por el vector de características $X$, se controla únicamente por la probabilidad de ser tratado $\mathrm{p}(\mathrm{X})^{4}$, lo que acaba con el problema de dimensionalidad.

La nueva expresión para el efecto promedio del tratamiento sobre los tratados, haciendo uso del propensity score será:

$$
\operatorname{ATT}(X)=E\left(y_{1} \mid D=1, p(X)\right)-E\left(y_{0} \mid D=0, p(X)\right)
$$

El cálculo de propensity score consiste en la estimación de un modelo de elección discreta para modelar la variable de participación en el programa, condicional a un vector de características individuales que puede haber influido en dicha probabilidad. Para este propósito pueden emplearse los modelos Probit y Logit.

\subsubsection{Estimador Matching no paramétrico}

La principal ventaja de los estimadores de Matching no paramétricos, es que a diferencia de los métodos paramétricos, no requieren especificar una forma funcional para la ecuación de resultados, lo que evita la posibilidad de sesgo por errores de especificación (Petra, 2008).
Después de estimado el propensity score, se calcula el estimador Matching $\hat{\mathrm{a}}^{\mathrm{M}}$ del efecto promedio del tratamiento sobre los tratados ATT, promediando sobre la región de soporte común ${ }^{5} \mathrm{~S}$, la diferencia entre los resultados de los individuos tratados y no tratados con propensity score cercanos, usando factores ponderadores $w$ :

$$
\hat{\alpha}^{M}=\frac{1}{n_{T} \in S} \sum_{i \in\{D=1, S\}}\left[Y_{1 i}-\sum_{j \in\{D=0, S\}} w_{i, j} Y_{0 j}\right]
$$

Donde $\mathrm{n}_{\mathrm{T}}$ es el total de individuos tratados. El subíndice $i$ se refiere al individuo tratado, $j$ al individuo de control.

Es decir, para cada observación i de la muestra de individuos tratados, se conforma un promedio ponderado de observaciones del grupo de control para el individuo i mediante los factores ponderadores $w_{i j}$. Finalmente, los efectos del tratamiento individuales son promediados entre todos los tratados, para estimar así ATT.

El estimador de Matching no paramétrico más común es el del vecino más cercano, en el cual cada individuo del grupo de tratamiento se empareja con un individuo del grupo de control, de modo que sea el más cercano en términos del propensity score $p(X)$. Es decir, se escoge el individuo no tratado $j$ para ser el contrafactual del individuo $i$, de modo que formalmente, el grupo de control del individuo $i\left(C^{\circ}(i)\right)$, es sólo un individuo $j$ que cumple:

$$
C(i)=\left\{j:\left\|p_{i}-p_{j}\right\|=\min _{k \in\{D=0, S\}}\left\|p_{i}-p_{k}\right\|\right\}
$$
modo.

De modo que $w_{(\mathrm{i}, \mathrm{k})}=1$, si $k=j$ y $k=0$ de otro

En el estimador del vecino más cercano, cada individuo del grupo de tratados tiene como contraparte a sólo uno de los individuos del grupo de control. Se puede ampliar el conjunto de comparación y mejorar la eficiencia de la estimación, si se incluyen otros individuos del grupo de control que sean también "similares" a los del grupo de tratamiento, donde lógicamente la

4. $\mathrm{p}\left(\mathrm{X}_{-} \mathrm{i}\right)$ es la probabilidad de que el individuo $i$ sea sujeto de tratamiento, definida como: $\mathrm{p}\left(\mathrm{X}_{i}\right)=\operatorname{Prob}\left(\mathrm{d}_{i}=1 \mid \mathrm{X}_{i}\right)=\mathrm{E}\left(\operatorname{di}_{i} \mid \mathrm{X}_{i}\right)$, donde: $\operatorname{0<p}\left(\mathrm{X}_{i}\right)<1$

5. La zona de soporte común corresponde a aquella zona en la cual los valores de $p(X)$ son comunes en ambas distribuciones, de tratados y de control. 
similitud está dada por la cercanía de sus valores de propensity score. Estos estimadores se conocen como Estimadores de Matching Suavizados Ponderados. Uno de los estimadores de este tipo más utilizados es el estimador Kernel. El estimador Matching de regresión Kernel escoge los pesos de tal forma que las observaciones del control que se encuentran más cerca del individuo del grupo de tratamiento, en términos de la distancia euclidiana, reciben una mayor ponderación:

$$
w_{i j}=\frac{K\left(\frac{p\left(X_{i}-X_{j}\right)}{h_{n}}\right)}{\sum_{k \in\{D=0\}} K\left(\frac{p\left(X_{i}-X_{j}\right)}{h_{n}}\right)}
$$

Siendo $K\left(\right.$.) la función Kernel ${ }^{6}$ y $h_{\mathrm{n}}$ el ancho de banda ${ }^{7}$ o parámetro de suavización.

\subsection{Estimación mediante variables instrumentales (VI)}

El método de variables instrumentales es utilizado en estudios no experimentales, con el propósito de controlar el sesgo de selección debido a factores no observables ${ }^{8}$. La técnica utiliza una o más variables que afectan la participación (instrumentos para la variable endógena), pero no los resultados de los participantes. Esto identifica la variación exógena en los resultados atribuibles al programa (Moral, 2009).

En el método de variables instrumentales, el instrumento para la variable endógena $Z$, debe cumplir con los supuestos:

i. $a_{-} i=a \quad \forall \_i$. Es decir, el efecto del tratamiento es homogéneo.

ii. $P(D=1 \mid X, Z) \neq P(D=1 \mid, X)$

iii. $E(U / Z)=E(U)$

Los supuestos $i$-iii permiten identificar el efecto del tratamiento:

$$
\begin{aligned}
& \gamma_{i}=\beta+a D_{i}+\mu_{i}(11) \\
& E\left(\gamma_{i} \mid Z_{i}\right)=\beta+a E\left(D_{i} \mid Z_{i}\right)+E\left(u_{i} \mid Z_{i}\right) \\
& E\left(y_{i} \mid Z_{i}\right)=\beta+a P\left(Z_{i}\right)+E\left(\mu_{i}\right)(12)
\end{aligned}
$$

6. La función Kernel que se utiliza habitualmente es la Kernel biponderada (Petra, 1999), la cual viene dada por:

$$
\begin{aligned}
K(s) & =\frac{15}{16}\left(s^{2}-1\right)^{2} \text { para }|s|<1 \\
& =0 \text { Para otros casos }
\end{aligned}
$$

7. El ancho de banda delimita la cantidad de individuos del grupo de control que se utilizan como contra factual de cada uno de los individuos tratados. Con valores altos de $h_{n}$ será más probable que $|s| \ll 1$ y exista una mayor cantidad de individuos del grupo de control para los cuales su ponderación $\mathbf{w} \neq \mathbf{0}$. En la literatura se recomiendan anchos de banda entre 0.2 y 0.4 .

8. Para advertir el problema, considérese el siguiente modelo que permite comparar los resultados de los individuos que participan en el programa, con aquellos que no participan:

$D_{i}=1$ si $d_{i} \geq 0 \quad d_{i}=g\left(X_{i}, v_{i}\right)$.

$$
\gamma_{i}=\beta+a_{i} D_{i}+\gamma X_{i}+\mu_{i}
$$

$D_{i}=o$ en otro caso.

$a_{i}=$ Efecto del tratamiento sobre el individuo $i$.

Donde $\mathrm{X}_{\mathrm{i}}$ es un vector de características observadas de los individuos que afecta sus resultados $\left(\mathrm{y}_{\mathrm{i}}\right)$ y $\gamma$ el vector que contiene sus coeficientes. La variable $\mathrm{d}_{i}^{*}$ es una variable latente, cuyo valor es función de las características observadas $\mathrm{X}_{\mathrm{i}} \mathrm{y}$ de componentes no observados $\mathrm{v}_{\mathrm{i}}$. Si el componente no observado afecta además de la asignación del tratamiento, los resultados de los individuos, $\mathrm{y}_{\mathrm{i}}$, existirá correlación entre el componente no observado y el error de la ecuación de resultados $\mathrm{u}_{\mathrm{i}}$ : $\operatorname{Cov}\left(v_{i}, \mu_{i}\right) \neq 0$.

Como la asignación del tratamiento depende de características observables y no observables, entonces $\mathrm{E}\left(\mathrm{D}_{\mathrm{i}} \mid \mathrm{X}_{\mathrm{i}}, \mathrm{v}_{\mathrm{i}}\right) \neq \mathrm{E}\left(\mathrm{D}_{\mathrm{i}} \mid \mathrm{X}_{\mathrm{i}}\right)$, lo que sumado a la correlación entre el componente no observado y el error de la ecuación de resultados $\mu_{i}$ causa la endogeneidad de la variable de tratamiento $D_{i}: \operatorname{Cov}\left(\mu_{i}, D_{i}\right) \neq 0$. 
En el modelo de la ecuación (11) se omite el vector de características $X_{i}$ por simplicidad. Cuando el instrumento para la variable endógena $Z$ toma dos valores diferentes $Z^{\star}$ y $Z^{\star \star}$ se puede identificar a a partir de (12):

$$
\alpha=\frac{E\left(y_{i} \mid Z_{i}=Z^{*}\right)-E\left(y_{i} \mid Z_{i}=Z^{* *}\right)}{P\left(Z^{*}\right)-P\left(Z^{* *}\right)}
$$

La ecuación (13) revela que la identificación de $\mathbf{Z}_{i}$ debe causar la suficiente variación en el propensity score, de lo contrario, el denominador será muy pequeño, lo que conduce a un estimador impreciso del efecto del tratamiento (Blundell y Costa, 2007).

Los pasos para la estimación de a son:

i. Estimar el modelo de respuesta binaria $P(D=1 \mid X, Z)$, a partir de una regresión Probit 0 Logit de $D_{i}$ sobre $X_{i}, Z_{i}$

ii. Estimar la ecuación: $y_{i}=\beta+a D_{i}+\gamma X_{i}+u_{i}$ por VI utilizando como instrumentos $X_{i}, \widehat{P} \nabla_{i}$. de la estimación por VI se obtiene el efecto estimado del tratamiento $\widehat{a}$.

La técnica de VI para estimar el efecto del tratamiento falla cuando el efecto del tratamiento es heterogéneo. Para incorporar la heterogeneidad del impacto del programa, el modelo de la ecuación (11) debe ser transformado:

$\gamma_{i}=\beta+a_{i} D_{i}+e_{i} \quad(14)$

$e_{\mathrm{i}}=\mu_{\mathrm{i}}+\mathrm{D}_{\mathrm{i}}\left(\mathrm{a}_{\mathrm{i}}-\mathrm{a}\right)$

Bajo heterogeneidad del efecto del tratamiento, el instrumento $Z$ no es exógeno en la ecuación de resultados (14), cuando se presenta selección sobre las ganancias idiosincrásicas $a_{i}-a^{9}$, porque:

$E(e \mid Z)=E(u \mid Z)+P(Z) * E\left(\alpha_{i}-\alpha^{A T E} \mid D=1, Z\right)$

Y para que $Z$ sea exógeno en la ecuación de resultados, se requiere que: $\mathrm{E}(e \mid \mathrm{Z})=\mathrm{E}(e)$, Lo cual solo se cumple si: $E\left(a_{i}-a^{A T E} \mid D=1, Z\right)=0$.

\subsection{Efecto Local Promedio del Tratamiento (LATE)}

El estimador LATE fue propuesto por Imbens y Angrist (1994), para identificar el impacto del tratamiento ante cambios locales en el instrumento $Z$, cuando el efecto del tratamiento es heterogéneo. Si el instrumento local $Z$ es una variable discreta, como una variable binaria, cuyo estado es influenciado por una determinada política, LATE mide el efecto del tratamiento sobre los individuos que cambian su status de tratamiento ante el cambio en el valor del instrumento (por efecto de la política).

El principio básico de LATE es que, bajo ciertas condiciones, el cambio en $Z$ reproduce localmente la asignación aleatoria al inducir a los individuos a alterar su status de participación sin alterar sus resultados potenciales $\gamma_{0}, \gamma_{1}$ (Blundell y Costa, 2007).

Siguiendo a Arrazola y Hevia (2003), sea $\gamma_{i}$ la variable sobre la cual se quiere medir el impacto de la política, ella dependerá, en general, tanto del valor de $D_{i}$ como de $Z_{i}$. Esta dependencia puede expresarse como $\gamma_{i}\left(D_{i}, Z_{i}\right)$. Adviértase que hay solo cuatro posibles resultados: $\gamma_{\mathrm{i}}(1,1), \gamma_{\mathrm{i}}(1,0), \gamma_{\mathrm{i}}(0,1), \mathrm{y}_{\mathrm{i}}(0,0)$. El Efecto Local de Tratamiento Promedio esta dado por:

$$
\mathrm{LATE}=\mathrm{E}\left[\gamma_{\mathrm{i}}(1,1)-\gamma_{\mathrm{i}}(0,0)\right](16)
$$

Angrist, Imbens y Rubin (1996) presentan y analizan los supuestos que deben verificarse para poder identificar y estimar el LATE mediante técnicas de Variables Instrumentales. Los supuestos los resume Wooldridge (2002):

$$
\text { i. Independencia: } \mathrm{Z}_{\mathrm{i}}^{\perp} \gamma_{\mathrm{i}} \text { (o), } \gamma_{\mathrm{i}}(1), \mathrm{D}_{\mathrm{i}}(1), \mathrm{D}_{\mathrm{i}} \text { (o) }
$$

En la presentación matemática del supuesto, el estatus de tratamiento depende de $Z_{\mathrm{i}}$, lo que puede expresarse como $D_{\mathrm{i}}\left(Z_{\mathrm{i}}\right)$. Así mismo, la variable resultado depende del estatus de tratamiento $D_{i}$, lo que puede ser expresado como $\gamma_{\mathrm{i}}\left(D_{\mathrm{i}}\right)$.

El supuesto requiere que el instrumento $Z$ sea tan bueno como si fuera asignado aleatoriamente, y que no afecte directamente los resultados.

$$
\text { ii. Monotonicidad: } D_{i}(1) \geq D_{i}(0)
$$

El supuesto busca descartar que $D_{i}(1)=0$ y $D_{i}$ (o)=1. Las unidades de la población que no satisfacen el supuesto de monotonicidad son llamados defiers.

9. Hay selección sobre las ganancias idiosincrásicas, cuando las ganancias idiosincrásicas a a-a y el componente no observable de la regla de selección $v$ están correlacionados. 
Aunque la técnica econométrica para la estimación de LATE es similar a la del estimador de variables instrumentales, LATE no representa ATT o ATE, solo mide el efecto del tratamiento en la subpoblación sobre la cual la política ejerce un cambio en la variable exógena $Z$, así que no es una medida representativa del grupo de tratados, ni de la población en general (Blundell y Costa, 2007).

\section{Datos}

El estudio pretende medir el impacto del crédito sobre las ganancias y la generación de empleo en las pequeñas y medianas empresas. Para este efecto, se utiliza la información disponible de la evaluación de impacto del Fondo Colombiano de Modernización y Desarrollo Tecnológico de las Micro, Pequeñas y Medianas Empresas (FOMIPYME) disponible en la página web del Departamento Nacional de Planeación.

FOMIPYME es uno de los instrumentos que ha creado el Gobierno Colombiano como parte de su política de apoyo empresarial, promoviendo la adopción de tecnologías duras y blandas, para ayudar a las MIPYME en el desarrollo de una estructura productiva más sólida y en la mejora de su posición competitiva en los mercados. FOMIPYME inició sus operaciones en el año 2001. Aunque la información recolectada por la firma que desarrolló el estudio no tenía como objetivo la evaluación del microcrédito, la misma contiene información suficiente de las empresas que permite la estimación del impacto del microcrédito sobre las variables de interés.

La información disponible en la base de datos de la evaluación de FOMIPYME y que se empleó en esta investigación es la siguiente: ganancias, número de trabajadores, sector de las firmas, antigüedad, experiencia del gerente, habilidad del gerente, número de productos ofrecidos, origen del préstamo, finalidad del crédito y si este es producto de la participación de la empresa en FOMIPYME.

La pregunta número 608 del formulario de FOMIPYME aporta la información clave que posibilita medir el efecto del microcrédito a partir de una encuesta no diseñada con este propósito:

¿Desde enero de 2002 a la fecha, esta empresa ha tenido algún tipo de préstamos?

La pregunta 604 del mismo formulario permite determinar cuál es el origen de los prés- tamos: i. Entidades financieras como bancos, corporaciones de ahorro y crédito, ii. Fondos de empleados o de asociaciones, cooperativas o fundaciones como las que realizan microcrédito, iii. Proveedores, iv. Prestamistas, v. Amigos o familiares.

La pregunta 606 estuvo dirigida a establecer cuál es el uso que se le dio a tales préstamos: i. Pago de alguna necesidad familiar, ii. Capital de trabajo (compra o alquiler de maquinaria, pagos de sueldos, compras de insumos, compras o alquiler de herramientas). iii. Compras de construcciones como casas, edificios, bodegas. iv. Pago de otras deudas.

Para las variables ganancias y número de trabajadores sobre las cuales se mide el impacto del microcrédito, la base de datos incluye información para los años 2001 y 2005. Esta información permite medir el impacto del microcrédito sobre los cambios en las ganancias y en número de trabajadores en este periodo de tiempo como resultado del microcrédito.

La muestra de FOMIPYME, contiene $652 \mathrm{em}$ presas beneficiarias ( 510 micros y 142 Pymes) y 188 empresas no beneficiarias (164 micros y 24 Pymes). Sin embargo, este estudio utiliza sólo el subconjunto de empresas beneficiarias, porque no está disponible la información sobre acceso al crédito para las empresas no beneficiarias.

\section{Resultados}

La estimación de los impactos del microcrédito se lleva a cabo mediantes las técnicas del Estimador Matching (bajo el supuesto de selección sobre los observables); Variables Instrumentales (suponiendo componentes no observados afecten tanto la selección del tratamiento como los resultados); y el estimador LATE, que conserva la propiedad de insesgamiento, así el efecto del tratamiento sea heterogéneo.

Para la variable de tratamiento se emplean tres alternativas diferentes:

i. Tomó un crédito que se utilizó para invertir en el negocio: Capital de trabajo o compra de inmuebles. La variable se designó $C X N$.

ii. Tomó un crédito y la decisión de solicitarlo tuvo que ver con la participación en FOMIPYME. La variable se designó TXF. 
iii. El crédito se utilizó para invertir en el negocio, y el crédito proviene de una fuente formal como entidades financieras y fundaciones que realizan Microcrédito. La variable se designó TRATA.

En los diagramas de barras de la gráfica 6 se compara la cantidad de individuos del grupo de tratados, con el número de individuos del grupo de control, para cada uno de los tratamientos.

\section{Grafica. 6. Número de tratados y controles}

\section{para cada tratamiento}

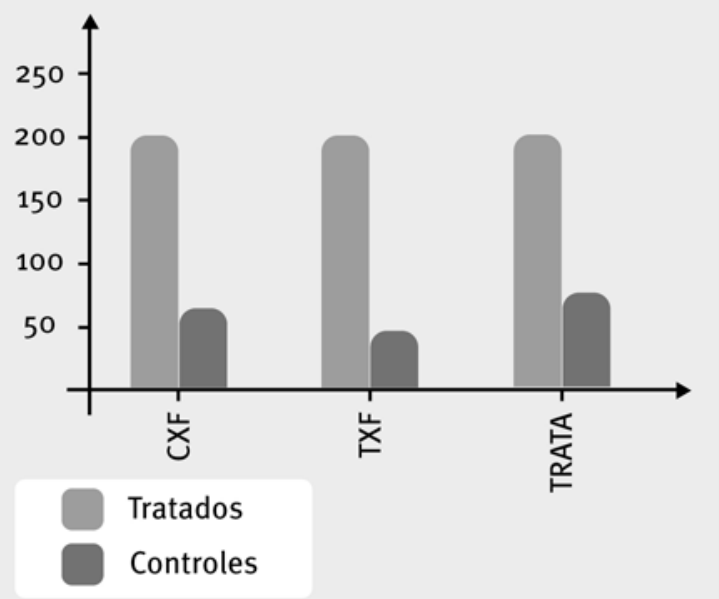

Fuente: Elaboración propia.

\subsection{Diferencias en Diferencias Matching}

Como ya se comentó, la base de datos de la FOMIPYME incluye información para los años 2001 (antes del tratamiento) y 2005 (después de tratamiento). La presencia de datos longitudinales de observaciones repetidas, permite evaluar el impacto del tratamiento sobre el cambio en las ganancias $(\Lambda G)$ y la generación de empleo de las Pymes $(\Lambda \mathrm{L})$, como resultado del acceso al crédito. Esté técnica es conocida como Diferencias en diferencias Matching. El estimador Matching de Diferencias en Diferencias es idóneo cuando existen dudas sobre la selección de la muestra (Blundell y Costa Dias, 2002).

Los resultados de las estimaciones Matching del vecino más cercano y Kernel, del efecto promedio del tratamiento sobre los tratados $\mathrm{a}^{\mathrm{ATT}}$, para cada variable de tratamiento considerada (CXN, TXF, TRATA), se resumen en la tabla 1 que aparece a continuación (Tabla 1).

La estimación del propensity score para cada variable de tratamiento considerada, aparece en el anexo 1. El algoritmo de estimación verifica, que al interior de cada intervalo, el promedio del propensity score entre los tratados y los de control no difieran. Además, se chequea que la distribución de las variables observables sea la misma, para los individuos tratados y para los no tratados.

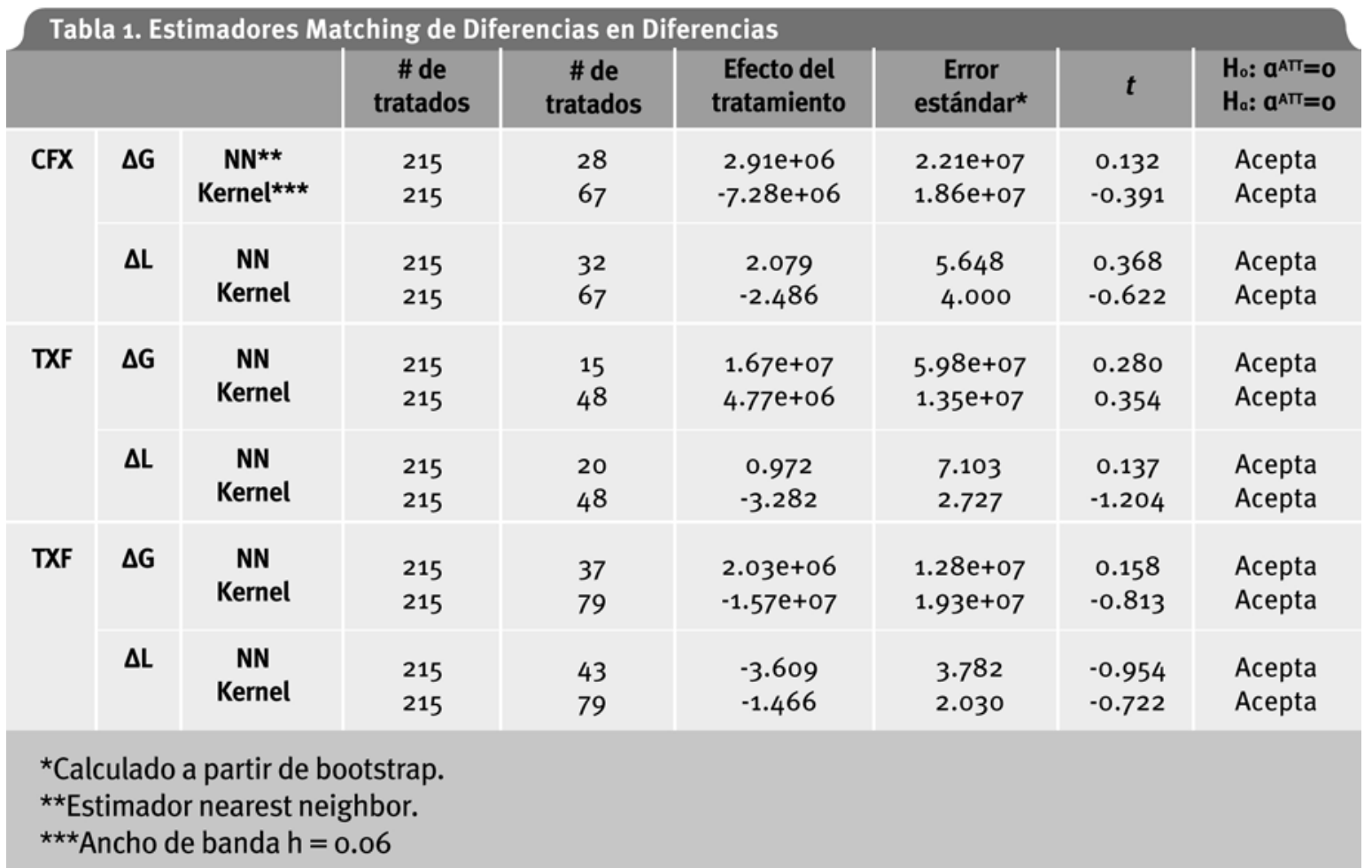


Las variables explicativas del modelo logit para la estimación del propensity score, son las siguientes:

$d S_{i}$ : Variables binarias de sector. $d S_{i}=1$ si la PYME Eal sectori, o en otro caso. Los sectores son: Industria, comercio, actividades inmobiliarias, agricultura, construcción, educación, hoteles y restaurantes, intermediación financiera, servicios, transporte.

Exper: Años de experiencia del gerente.

Habil: Variable indicadora que mide la capacidad del gerente, propietario o director de la empresa para negociar, asumir riesgos, prevenir y solucionar problemas, aprender de experiencias.

a: Antigüedad de la empresa.

dnp: Cambio en el número de productos que ofrece la empresa entre 2001 y 2005 . Variable binaria. dnp=1, si hay cambio, $o$ en otro caso.

El bajo poder explicativo de los modelos logit (pseudo Rcuadrado, 0.0236 del modelo de propensity score para la variable dependiente TRATA, 0.0374 del modelo de propensity score para la variable dependiente CXN, 0.0331 del modelo de propensity score para la variable dependiente TXF), podría explicarse por el hecho de que el grupo de control ha sido creado de manera minuciosa. Adviértase que en el caso de un experimento social, con asignación aleatoria del tratamiento, ninguna variable observable podría explicar la asignación al mismo por definición. En este sentido, esto es una medida indirecta del grado de efectividad de los grupos de control. Sin embargo, no se descarta que la diferencia entre los grupos de control y el de beneficiarios sea explicada principalmente por variables no observables no incluidas en el modelo.

Aunque los modelos no sirvan para explicar en gran medida la participación en los programas, si permiten definir los conjuntos de comparación de cada individuo del grupo de beneficiarios de los programas, para la estimación no paramétrica de los efectos de los tratamientos. La inclusión de variables con bajo poder explicativo en la estimación del propensity score busca mejorar la calidad del emparejamiento entre tratados y controles.

Observando los resultados del estadístico $t$, de los estimadores del vecino más cercano y Kernel, con más de 100 grados de libertad, para los cuales la distribución t student es aproximadamente igual a 1,66 , con $a=5 \%$, se acepta en todos los casos la hipótesis nula. Se concluye que el crédito formal, cualquier tipo de crédito, o el derivado de la participación en un programa de apoyo empresarial como FOMIPYME, ninguno tiene impacto positivo, sobre las ganancias de las firmas, o la generación de empleo.

\subsection{Estimación bajo selección sobre componentes no observados}

Si el componente no observado es un efecto fijo (constante en el tiempo), el mismo puede ser eliminado tomando primeras diferencias ${ }^{10}$. Esto es posible, dado que está disponible la información de las ganancias y número de empleados de las firmas, antes y después del tratamiento. Las variables presentes en el vector $X_{i}$ permanecen relativamente constantes (como la habilidad del gerente), o $\Lambda \mathrm{X}_{\mathrm{i}}$ toma los mismos valores para todas las firmas (cambio en los años de experiencia del gerente o antigüedad de las firmas). Por tanto, estas variables aparecen en la ecuación en primeras diferencias como un intercepto ${ }^{11}$. La variable cambio en el número de productos ofrecidos por la firma dnp no resultó estadísticamente significativa.

De las estimaciones de la regresión en primeras diferencias, que aparecen en el anexo 2, se infiere que el crédito, medido por las variables (CXN,TXF,TRATA), no tiene ningún efecto sobre las ganancias de las firmas o sobre la generación de empleo, porque en todos los casos se acepta la hipótesis nula, de que los coeficientes de la variable de tratamiento son cero, con una significancia de $a=5 \%$.

10. Adviértase que: $\left\{\begin{array}{l}y_{i t}=\beta+\alpha_{i} D_{i}+\gamma X_{i t}+u_{i t} \\ y_{i t-1}=\beta+\gamma X_{i t-1}+u_{i t-1}\end{array} \Rightarrow \Delta y_{i t}=\alpha_{i} D_{i}+\gamma \Delta X_{i t}+\Delta u_{i t}\right.$

11. Obsérvese que si $\Delta X_{i t}=c \Rightarrow \Delta y_{i t}=\alpha_{i} D_{i}+\gamma * c+\Delta u_{i t}=\Delta y_{i t}=\delta+\alpha_{i} D_{i}+\Delta u_{i t}$. Donde $\delta=\gamma * c$ 
Si el componente no observado, no es un efecto fijo, no se obtiene ganancia alguna con la diferenciación, y la estimación de los efectos de los tratamientos puede hacerse mediante variables instrumentales. Como instrumento para la variable endógena de tratamiento, se utilizó la variable ahorro de la firma s, que toma el valor de 1 si la firma guardaba algún dinero en alguna institución financiera, fundación o fondo de empleados, o en otro caso. Se supone que en el primer caso, es más fácil para la firma el acceso al crédito.

En la estimación por variables instrumentales de la ecuación (11), las variables agrupadas en $X_{-} \mathrm{i}$ no resultaron significativas, entonces sus resultados (salidas), son las mismas que las del estimador LATE. Los resultados aparecen en el anexo 3 . Si se asume que el efecto del tratamiento es homogéneo, el coeficiente de la variable de tratamiento debe interpretarse como el estimador de los efectos de los tratamientos (CXN,TXF,TRATA). Si se asume el efecto del tratamiento como heterogéneo, debe interpretarse como un estimador LATE.

\section{Conclusiones}

En este documento se mide el efecto del microcrédito, del crédito de cualquier origen, y del crédito derivado de la participación en FOMIPYME, sobre las ganancias y la generación de empleo de las PYME.

La evaluación del impacto de una política pública o programa como el microcrédito, está destinada a determinar si la intervención tuvo los efectos deseados sobre los individuos, y si los efectos son atribuibles al programa. La evaluación del impacto se realiza mediante inferencia causal, que consiste en determinar los efectos de la intervención, sobre algunas variables relevantes relacionadas con la política.

La principal complicación para la evaluación del impacto, es denominada problema fundamental de los estudios de causalidad, que consiste en el hecho de que un mismo individuo no puede recibir y no recibir tratamiento al mismo tiempo. Para superar este problema, las técnicas para la evaluación del impacto hacen uso de dos grupos. Un grupo afectado por el tratamiento (grupo de tratados) y un grupo de no tratados (grupo de control).
Cuando el estatus de tratamiento y la variable resultado, son afectados sólo por características observables disponibles para el investigador, es posible estimar los efectos del tratamiento, utilizando la técnica de emparejamiento, que como otras, emplea el supuesto de selección sobre los observables, para evitar el problema de sesgo de selección.

Bajo sesgo de selección, el efecto del tratamiento calculado a partir de la información disponible, es un estimador sesgado del efecto promedio del tratamiento sobre los tratados.

La técnica Matching ofrece una forma de construir un grupo de control lo más similar posible al grupo de tratados, en términos de las características observables antes de asignado el tratamiento. Los estimadores del vecino más cercano y Kernel son dos estimadores de emparejamiento, que utilizan el propensity score para escoger los contrafactuales del grupo de control, dentro del grupo de tratados.

Los estimadores Kernel y del vecino más cercano de ATT, del efecto del crédito formal, del crédito de cualquier origen, y del crédito derivado de la participación en FOMIPYME, sobre las ganancias y la generación de empleo de las PYME no resultaron significativos.

Si existen componentes no observados, que afecten tanto el estatus de tratamiento, como los resultados, no se puede trabajar bajo selección sobre los observables, y el estimador de mínimos cuadrados ordinarios, del efecto promedio del tratamiento sobre los tratados, a la ecuación (11), será un estimador sesgado. Para mitigar el problema, se puede recurrir al estimador de variables instrumentales, que es un estimador consistente de a. Sin embargo, si el efecto del tratamiento es heterogéneo, no se cumple el supuesto de exogeneidad del instrumento en el estimador IV.

Cuando el instrumento para la variable endógena es una variable binaria, cuyo estatus es afectado por una política pública, el estimador IV de la ecuación (11) sin las variables explicativas $X_{-} i$, es denominado efecto local promedio del tratamiento. LATE mide el efecto del tratamiento sobre los individuos que cambian su status de tratamiento, ante el cambio en el valor del instrumento por efecto de la política. 
El estimador del efecto del crédito formal, del crédito de cualquier origen, y del crédito derivado de la participación en FOMIPYME, sobre las ganancias y la generación de empleo de las PYME, obtenido mediante variables instrumentales, tampoco resultó significativo.

Como el instrumento para la variable endógena de acceso al crédito, que se utiliza en la estimación del efecto del tratamiento por IV de la ecuación (11), es la variable ahorro, el estimador LATE mide el efecto del microcrédito, sobre las firmas que cambian su estatus de tratamiento, al adoptar una cultura de ahorro, como resultado de una política diseñada para tal fin. El estimador LATE efecto del crédito formal, del crédito de cualquier origen, y del crédito derivado de la participación en FOMIPYME, sobre las ganancias y la generación de empleo de las MIPYME tampoco resultó significativo. De donde se deriva que la política de incentivar el ahorro de las firmas, para que mejoren su posibilidad de acceder al crédito, no tiene efectos sobre las ganancias de las firmas, o la generación de empleo.

La conclusión final de este artículo es que no se encontró ninguna evidencia estadística de que exista un efecto positivo del crédito formal (microcrédito), del crédito de cualquier origen, y del crédito derivado de la participación en programas de apoyo empresarial como FOMIPYME, sobre las ganancias y la generación de empleo de las MIPYME.

Estos resultados desalentadores, sobre los efectos del microcrédito estimados en esta investigación, pueden ser explicados por los hallazgos de Henríquez (2009), en el sentido de que lo que realmente mejora el desempeño de las empresas, no es recibir el microcrédito, sino su uso intensivo.

Por otra parte, el microcrédito tal vez sólo sea un instrumento que le permita a las MIPYME subsistir, y como se sostiene en Cotler y Rodríguez (2009), contribuya a la acumulación de activos físicos de los hogares, que como ellos mismos comentan, es un buen indicador de bienestar de los hogares.

Morduch (2009) sostiene que la mayoría de la gente que vive en la pobreza tiene una vida financiera incierta, porque sus ingresos fluctúan mucho de un día al otro, así que las personas están buscando el modo de gestionar mejor sus recursos, no necesariamente para que sus negocios prosperen, sino para sortear los vaivenes. En su opinión, tener acceso no sólo al crédito sino también al ahorro, seguros y envío de dinero proporciona beneficios a los pobres al ayudarles a estabilizar sus vidas. Aunque una cuenta de ahorro tal vez no saque a una persona de la pobreza, puede reducirle sus niveles de estrés y ayudarle a evitar una catástrofe financiera.

\section{Referencias}

Aedo, C. y González, P. (2002). Una evaluación paramétrica y no paramétrica del fondo de tierras y aguas indígenas. [Technical Report]. Santiago de Chile: Departamento de Ingeniería Industrial de la Facultad de Ciencias Físicas y Matemáticas de la Universidad de Chile.

Angrist, J., Inbens, G. y Rubin, D. (1996). Identification of causal effects using instrumental variables. Journal of the American Statistical Association 91(434) (pp. 444-455).

Arango, R. (2008). La incidencia de las microfi-nanzas en Colombia. El Cuaderno - Escuela de Ciencias Estratégicas 2(3) (pp. 69-81).

Aristizábal, R. (2007). El microcrédito como alternativa de crecimiento en la economía colombiana. Recuperado 16/05/2010 de: http://www.portalmicrofinanzas.org/gm/document-1.9.37392/Microcr\%C3\%Agdito\%20 Colombia\%20 2007.pdf.

Aroca, P. (2002). Microcrédito, evaluación de impacto. Casos: Brasil y Chile. Antofagasta. Chile: Instituto de Economía Aplicada Regional (IDEAR), Universidad Católica del Norte.

Arrazola, M. y De-Hevia, J. (2003). Evaluación económica de políticas educativas: Una ilustración con la Ley General de la Educación de 1970. Hacienda Pública Española. Revista de Economía Pública 164(1) (pp. 111-127).

Barona, B. (2004). Microcrédito en Colombia. Estudios Gerenciales 20(9o) (pp. 79-104).

Blundell, R. y Costa, M. (2007). Alternative approches to evaluation ineEmpirical microeconomics. Recuperado 12/02/2011 de: http://www.ucl.ac.uk/ uctp39a/BlundellCostaDias-27-09-2008.pdf. 
Blundell, R. y Costa, M. (2002). Alternative approaches to evaluation in empirical microeconomics. Londres, R.U.:Institute of Fiscal Studies, University College London.

Cárdenas, P. (2004). La banca comercial y las microfinanzas. Bogotá, Colombia: Asociación Bancaria y de Entidades Financieras de Colombia.

Cotler, P. y Rodríguez, E. (2009). Microfinanzas y bienestar del hogar en México. Recuperado 20/02/2011 de: http://www. itesm.mx/egap/que_es_egap/inv_pub/ EGAP_EC_09_06.pdf.

Dehejia, R. y Wahba, S. (1999). Causal effects in no nexperimental studies: reevaluating the evaluation of training programs. Journal of the American Statistical Association (94) (pp. 1053-1062).

Henríquez, F. (2009). Microcrédito y su Impacto: Un Acercamiento con Datos Chilenos. BID. Recuperado 15/03/2011 de http:// idbdocs.iadb.org/wsdocs/getdocument. aspx?docnum=2191152.

Holland, P. (1986). Statistics and causal inference. Journal of the American Statistical Association 81(369) (pp. 945-970).

Imbens, G. y Angrist, J. (1994). Identification and estimation of local average treatment effects. Econometrica 62(2) (pp. 467-75).

Kirkpatrik, C. y Maimbo S.M. (2002). The Implications of the evolving microfinance agenda for regulatory and supervisory policy. Development Policy Review 20(3) (pp. 293-304).

Larraín, C. (2009). ¿Existe un modelo de microfinanzas en América Latina? Santiago, Chile: CEPAL.

Marbán, R. (2005). Evolución y perspectivas de futuro del nuevo sistema de microcréditos desarrollado e implantado por el Grameen Bank en Bangladesh. Ponencia de la VII Reunión de Economía Mundial en la Universidad Complutense de Madrid. Madrid, Epaña.

Mena, B. (2001). Microcréditos: un medio efectivo para el alivio de la pobreza. Recuperado 15/01/2011 de: http://www.rimisp. org/ getdoc.php?docid=2250.

Microfinance Information Exchange. (2009. Primera aproximación al mercado microfinanciero. Recuperado 20/03/2011 de: http://www.themix.org/sites/default/ files/Co-lombia\%2oMicrofinance $\% 20$ Sector\%20Analy -sis\%202009.pdf.

Moral, I. (2009). Técnicas cuantitativas de evaluación de políticas públicas. Curso de evaluación de políticas públicas y programas presupuestarios. Recuperado 16/01/2011 de: http://www.sefin.fortaleza.ce.gov. br/apresentacoes/gerados/apresentacoes_madri/Ignacio_Moral.pdf

Morduch, J. (2009). Portfolios of the Poor: How the World's Poor Live on \$2 a Day. Princenton, USA: Princeton University Press.

Norton, R. (2004). Política de desarrollo agrícola, conceptos y principios en capacitación en políticas agrícolas y alimentarias. Roma, Italia: FAO 2. (pp. 337-436).

Todd, P. (1999). A practical guide to implementing matching estimators. Guide prepared for IADB meeting in Santiago. Recuperado 07/02/2011 de: http://athena.sas.upenn. edu/ petra/papers/prac.pdf.

Todd, P. (2008). Matching estimators. En: The New Palgrave Dictionary of Economics (2a ed). Steven N. Durlauf and Lawrence E. (Eds). Blume, Reino Unido: Palgrave MacMillan.

Rosenbaum, P. y Rubin, D. (1983). The central role of the propensity score in the observational studies for causal effects. Biometrika 70(1) (pp. 41-55).

Wooldridge, J. M. (2002). Econometric Analysis of Cross Section and Panel Data. Cambridge, USA: MIT Press. 
Anexo 1.

Propensity Score TRATA

ogistic regression

$\begin{array}{ccr}\text { Number ot obs } & & \\ \text { LR chi2(9) } & = & 7.65 \\ \text { Prob }>\text { chi2 } & = & 0.5702\end{array}$

og likelihood $=-\mathbf{1 5 8 . 1 8 1 1 2}$

Pseudo R2 $=0.0236$

\begin{tabular}{r|rrrrrr}
\hline trata & Coef. & std. Err. & \multicolumn{1}{c}{$z$} & $\mathrm{P}>|\mathrm{z}|$ & \multicolumn{2}{c}{ [95\% Conf. Interval] } \\
\hline ds1 & -.0784423 & .7007757 & -0.11 & 0.911 & -1.451937 & 1.295053 \\
ds2 & -.5770193 & .7857952 & -0.73 & 0.463 & -2.11715 & $\mathbf{9 6 3 1 1 0 9}$ \\
ds3 & -.979517 & 1.030835 & -0.95 & 0.342 & -2.999917 & 1.040883 \\
ds5 & -.330949 & 1.082695 & -0.31 & 0.760 & -2.452993 & 1.791095 \\
ds7 & -5409359 & .8491698 & 0.64 & 0.524 & -1.123406 & 2.205278 \\
ds10 & -1.203963 & 1.229741 & -0.98 & 0.328 & -3.614211 & 1.206285 \\
a & .0079679 & .0143792 & 0.55 & 0.579 & -.0202148 & .0361506 \\
exper & -.0172382 & .0136827 & -1.26 & 0.208 & -.0440558 & .0095794 \\
habi1 & .1284824 & .1148632 & 1.12 & 0.263 & -.0966453 & .3536101 \\
ccons & .4361816 & 1.062574 & 0.41 & 0.681 & -1.646426 & 2.518789 \\
\hline
\end{tabular}

ote: the common support option has been selected

he region of common support is $[.446227, .8755183]$

\section{Propensity Score CXN}

igistic regression

Number of obs=

$\begin{array}{llr}\text { LR chi2 }(10) & = & 16.65 \\ \text { Prob chi2 } & = & 0.0825 \\ \text { Pseudo R2 } & = & 0.0374\end{array}$

ig likelihood $=-214.25847$

Pseudo R2 =

\begin{tabular}{|c|c|c|c|c|c|c|}
\hline cxn & coef. & Std. Err. & $z$ & $P>|z|$ & [95\% Conf. & erval] \\
\hline $\begin{array}{r}\text { ds1 } \\
\text { ds2 } \\
\text { ds3 } \\
\text { ds5 } \\
\text { ds7 } \\
\text { ds9 } \\
\text { ds10 } \\
\text { a } \\
\text { exper } \\
\text { habi } 1 \\
\text { _cons }\end{array}$ & $\begin{array}{r}.4445511 \\
.0720718 \\
-6521196 \\
-1.442916 \\
-.3734704 \\
-.5430139 \\
-1.036415 \\
.011744 \\
.0095368 \\
-1355366 \\
-1.731795\end{array}$ & $\begin{array}{r}.6144098 \\
.6956775 \\
.9773889 \\
1.239537 \\
.7111441 \\
1.369597 \\
1.278191 \\
.0121334 \\
.0121794 \\
.1040485 \\
.9679004\end{array}$ & $\begin{array}{r}0.72 \\
0.10 \\
0.67 \\
-1.16 \\
-0.53 \\
-0.40 \\
-0.81 \\
0.97 \\
0.78 \\
1.30 \\
-1.79\end{array}$ & $\begin{array}{l}0.469 \\
0.917 \\
0.505 \\
0.244 \\
0.599 \\
0.692 \\
0.417 \\
0.333 \\
0.434 \\
0.193 \\
0.074\end{array}$ & $\begin{array}{r}-.7596699 \\
-1.291431 \\
-1.263528 \\
-3.872363 \\
-1.767287 \\
-3.227374 \\
-3.541623 \\
-.012037 \\
-.0143343 \\
-.0683947 \\
-3.628845\end{array}$ & $\begin{array}{r}1.648772 \\
1.435575 \\
2.567767 \\
.9865313 \\
1.020346 \\
2.141347 \\
1.468793 \\
.035525 \\
.0334079 \\
.3394678 \\
.1652546\end{array}$ \\
\hline
\end{tabular}

ite: the common support option has been selected

ie region of common support is [.15141799, .68482782]

\section{Propensity Score TXF}

Logistic regression

Log Tikelihood $=\quad-210.0385$

$\begin{array}{ccr}\text { Number of obs } & & 320 \\ \text { LR chi2(9) } & = & 14.38 \\ \text { Prob > chi2 } & = & 0.1094 \\ \text { Pseudo R2 } & = & 0.0331\end{array}$

\begin{tabular}{r|rrrrrr}
\hline txf & Coef. & std. Err. & \multicolumn{1}{c}{$z$} & P $|z|$ & [95\% Conf. Interva1] \\
\hline ds1 & .5625901 & .6350805 & 0.89 & 0.376 & -.6821449 & 1.807325 \\
ds2 & .2831881 & .7133972 & 0.40 & 0.691 & -1.115045 & 1.681421 \\
ds3 & .3405105 & .9873677 & 0.34 & 0.730 & -1.594695 & 2.275716 \\
ds5 & -.2758372 & 1.043592 & -0.26 & 0.792 & -2.32124 & 1.769566 \\
ds7 & -.7662409 & .7607172 & -1.01 & 0.314 & -2.257219 & .7247375 \\
ds10 & -.7665774 & 1.285579 & -0.60 & 0.551 & -3.286265 & 1.75311 \\
a & .0022779 & .0121028 & 0.19 & 0.851 & -.0214432 & .025999 \\
exper & .0021204 & .01202 & 0.18 & 0.860 & -.0214383 & .0256791 \\
habi1 & -1107485 & .1056786 & 1.05 & 0.295 & -.0963778 & .3178747 \\
cons & -1.550383 & .9883676 & -1.57 & 0.117 & -3.487548 & .386782 \\
\hline
\end{tabular}

Note: the common support option has been selected

The region of common support is [.14055113, .52344347] 


\section{Anexo 2}

Regresión en primeras diferencias - efecto de CXN sobre las ganancias

Linear regression $\begin{array}{lll}\text { Number of obs }= & & 278 \\ \text { F }(1,276) & = & 0.58 \\ \text { Prob }>\text { F } & = & 0.4487 \\ \text { R-squared } & = & 0.0023 \\ \text { Root MSE } & = & 1.1 \mathrm{e}+08\end{array}$

\begin{tabular}{r|rrrrrr}
\hline \multirow{2}{*}{ DG } & Coef. & Rtd. Err. & S & P $>|t|$ & \multicolumn{1}{c}{ [95\% Conf. Interva1] } \\
\hline Cxn & $1.10 \mathrm{e}+07$ & $1.45 \mathrm{e}+07$ & 0.76 & 0.449 & $-1.75 \mathrm{e}+07$ & $3.95 \mathrm{e}+07$ \\
_cons & $1.28 \mathrm{e}+07$ & 7319788 & 1.75 & 0.081 & -1593683 & $2.72 \mathrm{e}+07$ \\
\hline
\end{tabular}

Regresión en primeras diferencias - efecto de TXF sobre las ganancias

Linear regression
Number of obs $=$
$F(1,27 \overline{6})=$
Prob $>F$
R-squared
Root MSE

$.72 \mathrm{e}+07$

Linear regression
Regresión en primeras diferencias - efecto de TRATA sobre las ganancias
Number of obs $=$ $F(1,499)=$ Prob $>F$
R-squared ROOt MSE 95\% Conf. Interva1]

$-1.69 e+07$ $4.40 e+07$ cons $1.35 e+07$
$1.23 e+07$ 1. $54 \mathrm{e}+07$ $0.88 \quad 0.381$ $-1109765$

\begin{tabular}{|c|c|c|c|c|c|c|}
\hline DG & coef. & $\begin{array}{l}\text { Robust } \\
\text { std. Err. }\end{array}$ & $t$ & $P>|t|$ & [95\% conf. & Interva1] \\
\hline $\begin{array}{l}\text { trata } \\
\text { _cons }\end{array}$ & $\begin{array}{r}-1.66 e+07 \\
-2980459\end{array}$ & $\begin{array}{r}2.72 e+07 \\
4249210\end{array}$ & $\begin{array}{l}-0.61 \\
-0.70\end{array}$ & $\begin{array}{l}0.543 \\
0.483\end{array}$ & $\begin{array}{l}-7.01 e+07 \\
-1.13 e+07\end{array}$ & $\begin{array}{r}3.69 \mathrm{e}+07 \\
5368089\end{array}$ \\
\hline
\end{tabular}

Regresión en primeras diferencias - efecto de CXN sobre el empleo

Linear regression

$$
\begin{array}{llr}
\text { Number of obs }= & & 327 \\
\text { F }(1,325) & 3.62 \\
\text { Prob }>\text { F } & = & 0.0580 \\
\text { R-squared } & = & 0.0101 \\
\text { Root MSE } & = & 14.528
\end{array}
$$

\begin{tabular}{|c|c|c|c|c|c|c|}
\hline d7 & Coef. & $\begin{array}{l}\text { Robust } \\
\text { std. Err. }\end{array}$ & $t$ & $P>|t|$ & [95\% conf. & Interval] \\
\hline $\begin{array}{r}\text { cxn } \\
\text { conss }\end{array}$ & $\begin{array}{r}2.945578 \\
.3333333\end{array}$ & $\begin{array}{l}1.548213 \\
1.263229\end{array}$ & $\begin{array}{l}1.90 \\
0.26\end{array}$ & $\begin{array}{l}0.058 \\
0.792\end{array}$ & $\begin{array}{l}-.1002053 \\
-2.151805\end{array}$ & $\begin{array}{l}5.991362 \\
2.818471\end{array}$ \\
\hline
\end{tabular}


Regresión en primeras diferencias - efecto de TXF sobre el empleo

Linear regression

$$
\begin{array}{crr}
\text { Number of obs } & & 327 \\
\text { F }(1, \quad 325) & = & 2.42 \\
\text { Prob }>\text { F } & = & \mathbf{0 . 1 2 1 0} \\
\text { R-squared } & = & 0.0064 \\
\text { Root MSE } & = & \mathbf{1 4 . 5 5 5}
\end{array}
$$

\begin{tabular}{r|cccccr}
\hline & \multicolumn{5}{|c}{ Robust } & \multicolumn{2}{c}{ [1 } & Coef. & std. Err. & $t$ & $\mathrm{P}>|\mathrm{t}|$ & \multicolumn{2}{c}{ [95\% Conf. Interval] } \\
\hline txf & $\mathbf{2 . 3 7 6 9 4 8}$ & $\mathbf{1 . 5 2 8 7 9 1}$ & $\mathbf{1 . 5 5}$ & $\mathbf{0 . 1 2 1}$ & $\mathbf{- . 6 3 0 6 2 7 7}$ & $\mathbf{5 . 3 8 4 5 2 3}$ \\
_cons & $\mathbf{. 6 9 0 7 2 1 6}$ & $\mathbf{1 . 1 8 3 3 2 1}$ & $\mathbf{0 . 5 8}$ & $\mathbf{0 . 5 6 0}$ & $\mathbf{- 1 . 6 3 7 2 1 4}$ & $\mathbf{3 . 0 1 8 6 5 8}$ \\
\hline
\end{tabular}

Regresión en primeras diferencias - efecto de TRATA sobre el empleo

\begin{tabular}{r|rrrrrr}
\hline \multirow{2}{*}{$\mathrm{d} 1$} & Coef. & Std. Err. & \multicolumn{1}{c}{$\mathrm{t}$} & $\mathrm{P}>|\mathrm{t}|$ & \multicolumn{2}{c}{ [95\% Conf. Interva1] } \\
\hline trata & -3.139322 & 1.852122 & -1.69 & 0.091 & -6.784897 & $\mathbf{5 0 6 2 5 1 9}$ \\
_cons & 3.702703 & 1.50247 & 2.46 & 0.014 & .7453579 & $\mathbf{6 . 6 6 0 0 4 7}$ \\
\hline
\end{tabular}

\section{Anexo 3}

Estimador de VI del efecto de CXN sobre las ganancias

Instrumental variables (2SLS) regression

$$
\begin{aligned}
& \text { Number of obs }=280 \\
& \begin{array}{ll}
\text { wald chi2 }(1) & 1 \text { ) } \\
\text { Prob }>\text { chi2 } & 0.02
\end{array} \\
& \text { R-squared } \\
& \begin{array}{lll}
\text { Root MSE } & = & 9.7 \mathrm{e}+\dot{0} 8
\end{array}
\end{aligned}
$$

\begin{tabular}{|c|c|c|c|c|c|c|}
\hline ganancias & coef. & $\begin{array}{l}\text { Robust } \\
\text { std. Err. }\end{array}$ & $z$ & $P>|z|$ & [95\% conf. & Interva1] \\
\hline $\begin{array}{c}\operatorname{txf} \\
\text { _cons }\end{array}$ & $\begin{array}{l}1.87 e+08 \\
4.39 e+07\end{array}$ & $\begin{array}{l}1.08 \mathrm{e}+09 \\
4.00 \mathrm{e}+08\end{array}$ & $\begin{array}{l}0.17 \\
0.11\end{array}$ & $\begin{array}{l}0.863 \\
0.913\end{array}$ & $\begin{array}{l}-1.93 \mathrm{e}+09 \\
-7.40 \mathrm{e}+08\end{array}$ & $\begin{array}{l}\text { 2. } 30 \mathrm{e}+09 \\
8.28 \mathrm{e}+08\end{array}$ \\
\hline
\end{tabular}

\begin{tabular}{r|rccccr}
\hline \multirow{2}{*}{ ganancias } & \multicolumn{5}{|c}{ Robust } \\
Coef. & Std. Err. & \multicolumn{1}{c}{$z$} & $P>|z|$ & \multicolumn{2}{c}{ [95\% Conf. Interva]] } \\
\hline CXn & $-1.20 \mathrm{e}+09$ & $9.68 \mathrm{e}+09$ & -0.12 & 0.902 & $-2.02 \mathrm{e}+10$ & $1.78 \mathrm{e}+10$ \\
_Cons & $6.44 \mathrm{e}+08$ & $4.27 \mathrm{e}+09$ & 0.15 & 0.880 & $-7.72 \mathrm{e}+09$ & $9.00 \mathrm{e}+09$ \\
\hline
\end{tabular}

Instrumented: cxn

Instruments: s

Estimador de VI del efecto de TXF sobre las ganancias

Instrumental variables (2SLS) regression

\begin{tabular}{lll} 
Number of obs $=$ & \multicolumn{1}{c}{280} \\
wald chi2( 1$)$ & 0.03 \\
Prob chi2 $=$ & 0.8625 \\
R-squared & $=$ & 0.0123 \\
Root MSE & $=$ & $7.7 e+08$
\end{tabular}

Instrumented: txf

Instruments: $\quad s$ 


\section{Anexo 3}

Estimador de VI del efecto de TRATA sobre las ganancias

Instrumenta7 variables (2SLS) regression

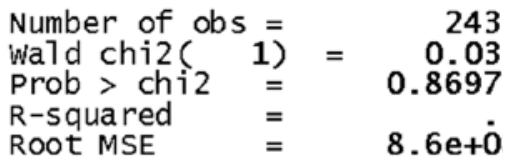

\begin{tabular}{r|rcrrrr}
\hline \multirow{2}{*}{ ganancias } & \multicolumn{5}{|c}{ Robust } \\
& cœf. & std. Err. & \multicolumn{1}{c}{$z$} & $P>|z|$ & \multicolumn{2}{c}{ [95\% conf. Interva]] } \\
\hline trata & $-4.31 \mathrm{e}+08$ & $2.63 \mathrm{e}+09$ & -0.16 & 0.870 & $-5.59 \mathrm{e}+09$ & $4.72 \mathrm{e}+09$ \\
-cons & $4.50 \mathrm{e}+08$ & $2.00 \mathrm{e}+09$ & 0.22 & 0.822 & $-3.47 \mathrm{e}+09$ & $4.37 \mathrm{e}+09$ \\
\hline Instrumented: & $\begin{array}{l}\text { trata } \\
\text { Ins truments: }\end{array}$ & & & & &
\end{tabular}

Estimador de VI del efecto de CXN sobre el empleo

Instrumental variables (2SLS) regression

$\begin{array}{llr}\text { Number of obs } & = & 327 \\ \text { wald chi2 } & \text { 1) } \\ \text { Prob chi2 }> & = & 0.01 \\ \text { R-squared } & = & 0.9345 \\ \text { Root MSE } & = & 55.876\end{array}$

\begin{tabular}{|c|c|c|c|c|c|c|}
\hline L & coef. & $\begin{array}{l}\text { Robust } \\
\text { std. Err. }\end{array}$ & $z$ & $P>|z|$ & {$[95 \%$ Conf. } & Interval] \\
\hline $\begin{array}{c}\text { cxn } \\
\text { cons }\end{array}$ & $\begin{array}{r}-46.18855 \\
46.72391\end{array}$ & $\begin{array}{l}562.1024 \\
252.2203\end{array}$ & $\begin{array}{r}-0.08 \\
0.19\end{array}$ & $\begin{array}{l}0.935 \\
0.853\end{array}$ & $\begin{array}{l}-1147.889 \\
-447.6189\end{array}$ & $\begin{array}{l}1055.512 \\
541.0667\end{array}$ \\
\hline
\end{tabular}

Instrumented: cxn

Instruments: s

Estimador de VI del efecto de TXF sobre el empleo

Instrumental variables (2SLS) regression

$\begin{array}{llr}\begin{array}{l}\text { Number of obs } \\ \text { wald chi2 }\end{array} & = & 327 \\ \text { Prob chi2 }>\text { chi2 } & = & 0.01 \\ \text { R-squared } & = & 0.9310 \\ \text { Root MSE } & = & 52.58 \dot{4}\end{array}$

\begin{tabular}{r|rrrrrr}
\hline & \multicolumn{5}{|c}{ Robust } \\
$\mathrm{L}$ & Coef. & Std. Err. & \multicolumn{1}{c}{$\mathrm{z}$} & $\mathrm{P}>|\mathrm{z}|$ & \multicolumn{2}{c}{ [95\% Conf. Interva1] } \\
\hline txf & $\mathbf{1 1 . 4 7 9 5}$ & $\mathbf{1 3 2 . 6 7 1 9}$ & 0.09 & $\mathbf{0 . 9 3 1}$ & $\mathbf{- 2 4 8 . 5 5 2 7}$ & 271.5116 \\
_cons & $\mathbf{2 1 . 2 9 1 2 1}$ & $\mathbf{5 4 . 6 0 9 1}$ & 0.39 & 0.697 & $\mathbf{- 8 5 . 7 4 0 6 6}$ & $\mathbf{1 2 8 . 3 2 3 1}$ \\
\hline
\end{tabular}

Instrumented: txf

Instruments: $\quad \mathrm{s}$

Estimador de VI del efecto de TRATA sobre el empleo

Instrumental variables (2SLS) regression

$\begin{array}{llr}\begin{array}{l}\text { Number of obs } \\ \text { Wald chi2 }\end{array} & = & 287 \\ \text { Prob chi2 } & = & 0.00 \\ \text { R-s quared } & = & 0.9475 \\ \text { Root MSE } & = & \mathbf{8 4 . 1 8}\end{array}$

\begin{tabular}{r|rrrrrr}
\hline & \multicolumn{5}{|c}{ Robust } \\
$\mathrm{L}$ & Coef. & Std. Err. & \multicolumn{1}{c}{$z$} & $\mathrm{P}>|\mathrm{z}|$ & \multicolumn{2}{c}{ [95\% Conf. Interva1] } \\
\hline trata & -129.9423 & $\mathbf{1 9 7 2 . 9 1}$ & -0.07 & 0.947 & -3996.774 & 3736.89 \\
_cons & 123.2115 & $\mathbf{1 4 6 3 . 5 8 9}$ & 0.08 & 0.933 & -2745.371 & 2991.794 \\
\hline
\end{tabular}

Instrumented: trata

Instruments: $s$ 